\title{
Are Gender Differences in Empathy Due to Differences in Emotional Reactivity?
}

\author{
Linda Rueckert, Brandon Branch, Tiffany Doan \\ Northeastern Illinois University, Chicago, USA. \\ Email: L-Rueckert@neiu.edu

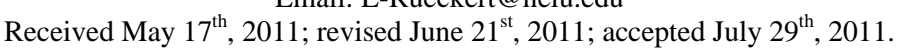

\begin{abstract}
The purpose of this study was to determine whether gender differences in empathy reflect differences in selfrated emotion, and whether they are influenced by the nature of the target of the empathy (friend or enemy). 24 men and 36 women were asked to rate how much happiness, sadness, and anger they would feel if each of ten scenarios happened to themselves, and how they would feel if it happened to a friend or enemy. Overall, women rated themselves as feeling more happiness and sadness than men, whether the event happened to themselves, or to a friend or enemy. This suggests gender differences in self-reported empathy may be due to differences in general emotional responsiveness. An empathy score was computed by subtracting, for each scenario, the rating for the other person from the rating for self. Women showed a greater difference between friend and enemy than men.
\end{abstract}

Keywords: Empathy, Sex Differences, Emotion

\section{Are Gender Differences in Empathy Due to Differences in Emotional Reactivity?}

In recent years there has been a dramatic increase in studies addressing empathy, the ability to feel and understand the thoughts and emotions of others. This has likely been driven at least in part by the growing field of social neuroscience and technological advances that allow us to study the neural basis of empathy to a much greater extent than was possible before.

The most common method of measuring empathy is selfreport questionnaires (e.g. Baron-Cohen \& Wheelwright, 2004; Davis, 1983; Mehrabian \& Epstein, 1972). One of the most robust and reliable results obtained from self-report studies is a gender difference, with women reporting greater empathy than men (e.g. Baron-Cohen \& Wheelwright, 2004; Davis, 1983; Eisenberg \& Lennon, 1983; Mehrabian, Young, \& Sato, 1988; Rueckert \& Naybar, 2008). However, this female superiority has not been found as often using other measures of empathy that are presumably more objective, leading Eisenberg and Lennon (1983) to suggest that the differences found using self-report may be due, at least in part, to demand characteristics. Evidence for demand characteristics have also been reported by others (Ickes, Gesn, \& Graham, 2000).

Nevertheless, there have been a few studies that reported similar gender differences using more objective measures. Dimberg and Lundquist (1990) found that women showed greater facial mimicry, as measured by electromyogram, to photos of emotional faces. Chapman, Baron-Cohen, Auyeung, Knickmeyer, Taylor, \& Hackett (2006) reported a negative correlation between fetal testosterone levels and empathy in children age 6 to 8 . In an event-related potential study, Fukushima and Hiraki (2006) found that women, but not men, exhibited medial-frontal negativity when their opponent in a game experienced a loss. Men exhibited this response only when they themselves experienced the loss.

Until very recently there were no neuroimaging studies of gender differences in empathy. In fact, a recent meta-analysis of fMRI studies of empathy for pain found no evidence for overall gender differences (Lamm, Decety, \& Singer, 2011). However, some studies have found significant gender differences under specific conditions. For example, Singer, Seymour, O’Doherty, Stephan, Dolan, \& Frith (2006) used functional MRI to measure brain activity while participants received mild electric shocks or witnessed a confederate receiving a similar shock. They manipulated participants' feelings toward the confederates by having them play a "prisoner dilemma" type of game. One of the confederates played the game fairly and the other played unfairly. They found that both men and women showed bilateral activation in pain-related areas of the brain (anterior insula and anterior cingulate) when they received a shock, and when they witnessed a "fair" confederate receive a shock. However, only women showed this activation when the "unfair" confederate was shocked. Results reported by Cheng, Chen, Lin, Chou, and Decety (2010) also support the idea that empathic responses may differ based on the target of the empathy. They found greater activation in the insula and anterior cingulate when participants saw hands and feet in painful positions and were asked to imagine that the picture depicted a loved one, compared to when they were asked to imagine that it depicted a stranger. However, they did not examine gender differences.

Many psychologists differentiate between emotional empathy, or the tendency to feel the same emotion as another person, and cognitive empathy, which is the knowledge and understanding of another person's thoughts and emotions, without necessarily feeling the same emotion (similar to "theory of mind”). An empathy scale developed by Davis (1983) yields separate scores for emotional empathy (labeled "emotional concern") and cognitive empathy (labled as "perspective taking”), and some studies have suggested that the gender difference may be limited to the emotional concern scale (Derntl et al., 2010). In the same study, Derntl et al. (2010) found that women showed greater activation than men in several brain regions, including the amygdala, across a number of empathy-related emotional judgment tasks. Interestingly, they found this sex difference in the brain despite equal performance by 
women and men on the tasks. Schulte-Rüther, Markowitsch, Shah, Fink, \& Piefke (2008) found that women showed greater activation in the right inferior frontal cortex and superior temporal sulcus, while men showed greater activation of the left temporoparietal junction, when rating their own and others' emotion. They interpreted this as greater emotional engagement on the part of their female participants.

Rueckert and Naybar (2008) utilized a chimeric faces task (Levy, Heller, Banich, \& Burton, 1983) to assess activation of the right cerebral hemisphere in normal participants. Although there were no differences in asymmetry found for the chimeric faces task, they found that right hemisphere activation correlated with self-reported empathy for women, but not for men. This suggests that women are more likely than men to recruit similar brain regions for empathy and for judgment of emotional expression.

Although most self-report scales do not differentiate between cognitive and emotional empathy, an examination of the items on these questionnaires suggests that they may assess predominantly emotional empathy. Furthermore, most of the items tend to focus on negative emotions, such as sadness (e.g. "It makes me sad to see a lonely stranger in a group"; Mehrabian \& Epstein, 1972). If, as has been suggested by a number of studies (e.g. Allen \& Markiewicz Haccoun, 1976; Blier \& Blier-Wilson, 1989) women tend to report higher levels of emotion than men, it's possible that what appears to be a gender difference in empathy is actually a difference in reported level of emotion. For example, a high rating to a statement such as "It makes me sad to see a lonely stranger in a group”, would result in a higher empathy score, although it could simply be due to a greater tendency to experience or report sadness in general.

The purpose of the present study was to examine gender differences in empathy in greater detail. Specifically, we sought to determine whether the difference might be due to levels of reported emotion. To do this we developed a new empathy scale that measured participants' self-reported emotion for events that occurred to themselves, as well as to other people. Because previous studies have suggested that empathy in men and women may vary depending upon the relationship between themselves and the target, we also varied this relationship. Participants were asked to rate how they would feel if the event happened to a good friend, and well as how they would feel if it happened to some one they did not like.

\section{Methods}

\section{Participants}

There were 60 participants (36 women and 24 men) who ranged in age from 19 to 47 , with a mean age of 24.5 years. The mean age for women (24.8 years) did not differ from the mean age for men (24 years). All participants signed an Informed Consent form that was approved by the Northeastern Illinois University (NEIU) Institutional Review Board.

\section{Materials}

In addition to the Informed Consent form and a short demographics form, all participants completed the following questionnaires:

Interpersonal Reactivity Index (IRI; Davis, 1983). This questionnaire consists of 28 statements that inquire about the thoughts and feelings of individuals in a variety of situations. It is a self-report questionnaire consisting of four 7-item subscales, each of which assesses a specific aspect of empathy. Scores for each item can range from 0 to 4 , with a higher score indicating a greater level of empathy. The The Perspective-Taking (PT) subscale assesses an understanding of the point of view of others (cognitive empathy). The Fantasy (FS) subscale assesses a physiological arousal to a filmed depiction of fictitious characters in movies, plays and books. The Empathetic Concern (EC) subscale assesses concern and sympathy for other people (emotional empathy). The Personal Distress (PD) subscale assesses feelings of personal anxiety in reaction to the emotions of others in tense social settings. The maximum score for each subscale is 28.

NEIU Empathy Scale. This scale was developed specifically for this study. It is comprised of ten scenarios (see Appendix). Participants are first asked to read through the scenarios and rate on a Likert scale from 1 to 5 the extent to which they would feel each of three emotions (happy, sad, and angry; $1=$ no emotion, 5 = extreme emotion). The scenarios were created with the expectation that they would yield an approximately equal number of happy, sad, and angry responses.

After rating how they would feel for all ten scenarios, participants were asked to go through all ten scenarios again and rate how they (the participant) would feel if each of the scenarios happened to their best friend (of the same gender) and to an enemy, or a person they do not like (of the same gender). Some of the scenarios were chosen with the expectation that they might be likely to yield different emotions depending on whether they happen to a friend or to an enemy (i.e. getting a ticket for parking in a handicapped space). All participants were first asked to rate how they would feel if the scenario happened to themselves. Half of them were then asked about the friend, followed by enemy, and the other half were asked about enemy followed by friend.

\section{Procedures}

After filling out the Informed Consent and demographics forms, participants were asked to fill out the IRI, followed by the NEIU Empathy Scale (half the participants filled them out in the opposite order).

\section{Results}

\section{IRI}

An ANOVA was conducted with gender as a between-subjects variable and subscale (EC, PT, PD, FS) as a repeated measure. There was a main effect of gender, $F(1.58)=$ 7.37, $p=.009, \eta^{2}=.11$. Men $(M=15.48,95 \%$ CI[14.28, 16.67] $)$ scored lower than women $(M=17.20,95 \%$ CI[16.5, 17.90]). This was modified by a trend toward a gender by subscale interaction, $F(3.174)=2.49, p=.062, \eta^{2}=.056$. Simple main effects post-hocs showed that the gender difference was significant only for EC ( $p=.001)$, and marginally significant for $\operatorname{PD}(p=.1)$.

\section{NEIU Empathy Scale}

A composite score for each of the three emotions (happy, sad, angry) was calculated for each participant under each condition (self, friend, enemy). Ratings were first analyzed to determine whether there were any differences in the strength of emotion reported by men and women. An ANOVA was conducted with gender as a between-subjects variable. Person (self, friend or enemy) and emotion (happy, sad, angry) were repeated meas- 
ures. There was a main effect of person, $F(2.116)=39.84, p$ $<.001, \eta^{2}=.41$. Tukey's HSD post-hoc analyses showed that emotional ratings for self were significantly higher than ratings for either friend or enemy $(p<.05)$. There was also a main effect of emotion, $F(2.116)=8.30, p<.001, \eta^{2}=.12$, that was modified by a person by emotion interaction, $F(4.232)=23.47$, $p<.001, \eta^{2}=.29$. Simple effects post-hoc analyses revealed that the main effect of person was significant only for sad and angry ( $p<.001$; see Figure 1$)$. There was also a trend toward an emotion by gender interaction $F(2.116)=2.57, p=.08, \eta^{2}=.04$. due to the fact that women gave higher ratings than men only for happy and sad (see Figure 2).

Empathy scores were calculated by identifying the predominant emotion for each participant in each of the ten scenarios. The predominant emotion was defined as the one that received the highest rating for the self. The friend empathy score is the difference between the rating given to the predominant emotion for self for that particular scenario, and the rating given to the friend for the same emotion on the same scenario. A composite friend empathy score was calculated by adding the difference scores for all ten scenarios. Thus, a higher score indicates a greater difference between the rating given to the self and the rating given to the friend, and therefore less empathy. Enemy empathy scores were calculated in the same manner using ratings given to the enemy. For this analysis the data was discarded for the four men and four women who rated only one emotion for each scenario. Spearman-Brown split-half reliability coefficients were at an acceptable level $(r=.80$ for both friend and enemy empathy scores).

An ANOVA was calculated with these empathy scores as the dependent variable. Gender was a between-subjects variable

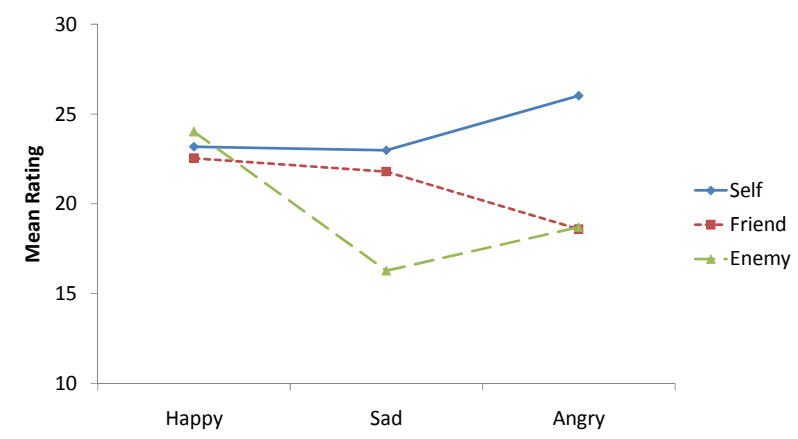

Figure 1.

Mean ratings for each of the three emotions given to the self, friend, and enemy on the NEIU scale.

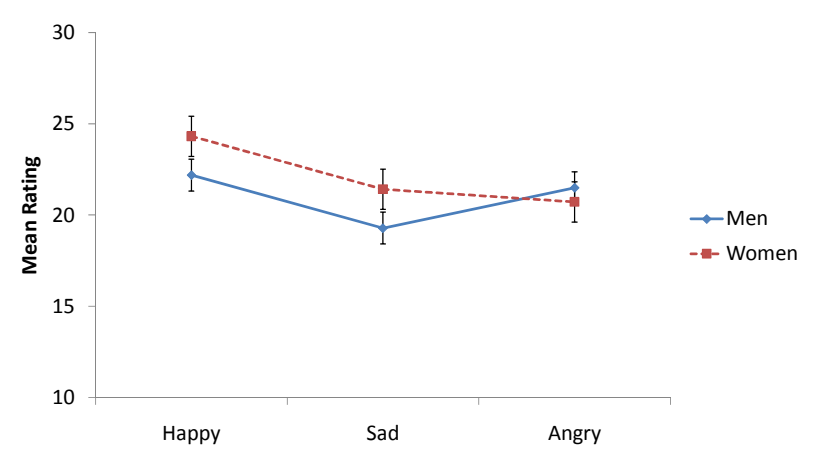

Figure 2.

Mean ratings given by men and women for each of the three emotions on the NEIU scale. Error bars indicate the standard error for the gender difference, pooled across emotions. and person (friend or enemy) was a repeated measure. There was a main effect of person, $F(1.58)=117.12, p<.001, \eta^{2}$ $=.67$. Empathy scores were lower (indicating greater empathy) for friends $(M=10.51,95 \% \mathrm{CI}[8.51,12.52])$ than for enemies ( $M=22.80,95 \%$ CI[20.44, 25.16]). This was modified by a gender by person interaction, $F(1.58)=9.22, p=.004, \eta^{2}=.14$. Figure 3 shows that, although both men and women showed greater empathy toward their friends than their enemies, the difference was greater for women.

\section{Relationship between Scales}

Neither empathy scores nor raw ratings on the NEIU scale correlated significantly with any of the four subscales of the IRI after a Bonferonni correction was applied. Ratings on the NEIU scale did correlate with each other: Overall ratings for self correlated with both friend ( $r=.77, p<.001)$ and enemy ratings ( $r$ $=.62, p<.001)$. These correlations were also significant within each gender (women: $r_{\text {self,enemy }}=.63, r_{\text {self,friend }}=.72$; men: $r_{\text {self,enemy }}=.69, r_{\text {self,friend }}=.82$, all $p$ s $<.001$ )

Item analysis. Detailed statistics for each of the ten scenarios (mean ratings for men and women for each item, etc.) can be found online at http://www.neiu.edu/ Iruecker/empathy.htm

\section{Discussion}

These results provide some limited support for gender differences in empathy but suggest that these differences are not ubiquitous, but, rather tend to occur under specific conditions. While we did replicate the common finding that women score higher on the IRI, the difference was only significant for EC (and marginally significant for PD). This is congruent with some previous studies that utilized the IRI (Derntl et al., 2010; Yang, Decety, Lee, Chen, \& Cheng, 2008). This result suggests that the gender difference may be limited to emotional empathy, and raises the possibility that it is due to differences in emotional reactivity. That possibility is also supported by the fact that, on the NEIU empathy scale, women reported that they would feel greater happiness and sadness when the scenario happened to themselves, as well as when it happened to others. This finding replicates others who have found that women report more intense ratings of happiness and sadness than men, but report less or equal levels of anger (Allen \& Markiewicz Haccoun, 1976; Blier \& Blier-Wilson, 1989).

The results of this study suggest that gender differences in empathy may reflect, at least in part, differences in emotional

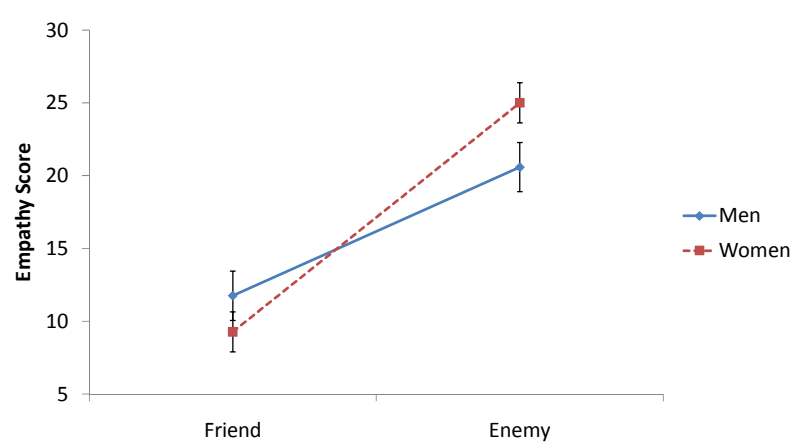

Figure 3.

Mean empathy scores (difference in ratings given to the self and other person) for friend and enemy on the NEIU scale. Error bars indicate the standard error for the gender difference. Note that a greater difference indicates less empathy. 
reactivity. This inference is also supported by the significant correlation between participants' ratings for themselves and for other people. However, in the empathy analyses overall ratings of emotional level were controlled on the NEIU scale by subtracting the emotional rating for others (friend and enemy) from that given for the self. Analyses of these scores did not show an overall gender difference in empathy, but revealed that women's level of empathy is more affected by the relationship they have with the other person; they showed somewhat greater empathy levels toward friends, but lower levels towards enemies. Although this result has not been reported before, it is congruent with results from studies of helping behavior. In a meta-analysis of studies measuring people's willingness to help in real-life situations Eagly and Crowly (1986) found that men were actually significantly more likely to provide aid than women. However, they pointed out that the vast majority of these studies involved providing help to a stranger, which women may find somewhat threatening. Some more recent studies have found no gender difference in less threatening situations (e.g. Reysen \& Ganz, 2006) or a tendency for women to give more help when the person receiving the help is a friend (George, Carroll, Kersnick \& Calderon, 1998).

The gender difference in empathy toward friends and enemies found in the present study appears to be at odds with results reported by Singer et al. (2006). Using fMRI, they found that women and men showed an equally strong empathic response in pain-related areas of the brain when they saw a "fair" confederate receive shocks. However, only women showed this response when they watched a confederate they believed had played a game unfairly receive shocks. There are numerous methodological differences between the two studies. In the study by Singer et al. both the fair and unfair confederate were unknown to the subjects. In the present study subjects were asked to think of a person who was known to them that they considered a friend or enemy. Thus, they actually had some type of relationship and previous interaction with the target. It is also possible that the vicarious experience of pain differs from empathy experienced in more complex emotional situations.

Most previous studies of empathy have not specified the target of the empathy, but have instead assessed it toward some generalized "other" person. The results of the present study suggest that the nature of the person to be empathized with is an important variable to consider. These results and those of other recent studies suggest that gender differences in empathy are more variable and context-dependent than has been suggested by the highly consistent results reported in earlier studies utilizing self-report. (For further discussion of contextual variables that may affect empathy, see de Vignemont \& Singer, 2006). Scores on self-report measures may reflect demand characteristics and willingness to admit to feelings of sadness, in addition to empathy. Of course, other measures of empathy also have limitations to their internal and external validity. A better understanding of empathy in general, and gender differences in particular, will require the utilization of a variety of methods under a variety of conditions.

\section{References}

Allen, J. G., \& Haccoun, D. M. (1976). Sex differences in emotionality: A multidimensional approach. Human Relations, 29, 711-722. doi:10.1177/001872677602900801

Baron-Cohen, S., \& Wheelwright, S. (2004). The Empathy quotient: An investigation of adults with Asperger syndrome or high functioning autism, and normal sex differences. Journal of Autism and Developmental Disorders, 34, 163-175.
doi:10.1023/B:JADD.0000022607.19833.00

Blier, M. J., \& Blier-Wilson, L. A. (1989). Gender differences in self-rated emotional expressiveness. Sex Roles, 21, 287-295. doi:10.1007/BF00289908

Chapman, E., Baron-Cohen, S., Auyeung, B., Knickmeyer, R., Taylor, K., \& Hackett, G. (2006). Fetal testosterone and empathy: Evidence from the Empathy Quotient (EQ) and the "Reading the Mind in the Eyes” test. Social Neuroscience, 1, 135-148. doi:10.1080/17470910600992239

Cheng, Y., Chen, C., Lin, C., Chou, K., \& Decety, J. (2010). Love hurts: an fMRI study. Neuroimage, 51, 923-929.

doi:10.1016/j.neuroimage.2010.02.047

Davis, M. H. (1983). Measuring individual differences in empathy: Evidence for a multidimensional approach. Journal of Personality and Social Psychology, 44, 113-126. doi:10.1037/0022-3514.44.1.113

de Vignemont, F., \& Singer, T. (2006). The empathic brain: how when and why? Trends in Cognitive Sciences, 10, 435-441. doi:10.1016/j.tics.2006.08.008

Derntl, B., Finkelmeyer, A., Eickhoff, S., Kellermann, T., Falkenberg, D., Schneider, F., \& Habel, U. (2010). Multidimensional assessment of empathic abilities: Neural correlates and gender differences. Psychoneuoendocrinology, 35, 67-82. doi:10.1016/j.psyneuen.2009.10.006

Dimberg, U., \& Lundquist, L. (1990). Gender differences in facial reactions to facial expressions. Biological Psychology, 30, 151-159. doi:10.1016/0301-0511(90)90024-Q

Eagly, A. H., \& Crowley, M. (1986). Gender and helping behavior: A meta-analytic review of the social psychological literature. Psychological Bulletin, 100, 283-308. doi:10.1037/0033-2909.100.3.283

Eisenberg, N., \& Lennon, R. (1983). Sex differences in empathy and related capacities. Psychological Bulletin 94, 100-131. doi:10.1037/0033-2909.94.1.100

Fukushima, H., \& Hiraki, K. (2006). Perceiving an opponent's loss: gender-related differences in the medial-frontal negativity. Social, Cognitive, and Affective Neuroscience, 1, 149-157. doi:10.1093/scan/ns/020

George, D., Carroll, P., Kersnick, R., \& Calderon, K. (1998). Gender-related patterns of helping among friends. Psychology of Women Quarterly, 22, 685-704. doi:10.1111/j.1471-6402.1998.tb00185.x

Ickes, W., Gesn, P. R., \& Graham, T. (2000). Gender differences in empathic accuracy: Differential ability or differential motivation? Personal Relationships, 7, 95-109. doi:10.1111/j.1475-6811.2000.tb00006.x

Lamm, C., Decety, J., \& Singer, T. (2011). Meta-analytic evidence for common and distinct neural networks associated with directly experienced pain and empathy for pain. NeuroImage, 54, 2492-2502. doi:10.1016/j.neuroimage.2010.10.014

Levy, J., Heller, W., Banich, M.T., \& Burton, L.A. (1983). Asymmetry of perception in free viewing of chimeric faces. Brain and Cognition, 2, 404-419. doi:10.1016/0278-2626(83)90021-0

Mehrabian, A., \& Epstein, N. (1972). A measure of emotional empathy. Journal of Personality, 40, 525-543. doi:10.1111/j.1467-6494.1972.tb00078.x

Mehrabian, A., Young, A. L., \& Sato, S. (1988). Emotional empathy and associated individual differences. Current Psychology: Research \& Reviews, 7, 221-240. doi:10.1007/BF02686670

Reysen, S. \& Ganz, E. (2006). Gender differences in helping in six U.S. cities. North American Journal of Psychology, 8, 63-68.

Rueckert, L., \& Naybar, N. (2008). Gender differences in empathy: The role of the right hemisphere. Brain and Cognition, 67, 162-167. doi:10.1016/j.bandc.2008.01.002

Schulte-Rüther, M., Markowitsch, H. J., Shah, N. J., Fink, G. R., \& Piefke, M. (2008). Gender differences in brain networks supporting empathy. NeuroImage, 42, 393-403. doi:10.1016/j.neuroimage.2008.04.180

Singer, T., Seymour, B., O’Doherty, J. P., Stephan, K. E., Dolan, R. J., \& Frith, C. D. (2006). Empathic neural responses are modulated by the perceived fairness of others. Nature, 439, 466-469. doi:10.1038/nature04271

Yang, C., Decety, J., Lee, S., Chen, C., \& Cheng, Y. (2009). Gender differences in the mu rhythm during empathy for pain: An electroencephalographic study. Brain Research, 1251, 176-184. doi:10.1016/j.brainres.2008.11.062 


\section{Appendix}

\section{NEIU Emotional Response Questionnaire}

The following 10 scenarios are to be used with each of the next 3 answer sheets. Please read the instructions at the top of each answer sheet carefully, because they are all different.

Scenarios:

1) You won $\$ 5,000$ in the lottery.

2) You bought a brand new pair of shoes two days ago. Today it started pouring rain as you were walking to the store and your shoes were ruined.

3) Your favorite team is going to the playoffs. You've got tickets. However, you can't get off work, and so will not be able to go.

4) You put money in to a vending machine to buy a candy bar, but the machine is broken so you lose your money.
5) You put money in to a vending machine to buy a candy bar. The machine malfunctions and gives you your money back, as well as giving you the candy bar.

6) You go to the grocery store to buy just one item. But you can't find any place to park legally, so you park in the handicapped space. When you come back 10 minutes later you have received a parking ticket.

7) You've been waiting in line for an hour to get tickets to a movie you really want to see. Just as you get up to the ticket window, they announce that they are sold out.

8) You're driving to work and you're late, so you start driving very fast. You accidentally run over a squirrel and kill it.

9) You tried out for a city-wide talent show and made it to the top five finalists.

10) As you're walking down the street a car drives by very fast through a puddle and splashes water all over you. 